\title{
The Influence of Surgical Experience on Postoperative Recovery in Fast-Track Bariatric Surgery
}

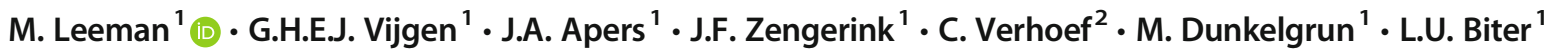

(C) Springer Science+Business Media, LLC, part of Springer Nature 2020

\begin{abstract}
Introduction Short duration of surgery is an important aspect in fast-track protocols. Peroperative training of surgical residents could influence the duration of surgery, possibly affecting patient outcome. This study evaluates the influence of the operator's level of experience on patient outcome in fast-track bariatric surgery.

Methods Data was analyzed of all patients who underwent a primary laparoscopic Roux-en-Y gastric bypass (LRYGB) or laparoscopic sleeve gastrectomy (LSG) between January 2004 and July 2018. Residents were trained according to a stepwise training program. For each operator, learning curves of both procedures were created by dividing the procedures in timesubsequent groups (TSGs). Data was also analyzed by comparing "beginners" with "experienced operators," with a cut-off point at 100 procedures. Primary outcome measure was duration of surgery. Secondary outcome measures were length of hospital stay (LOS), complications, and readmission rate within 30 days postoperatively.

Results There were 4901 primary procedures (53.1\% LSG) performed by seven surgeons or surgical residents. We found no difference between beginning and experienced operators in complications or readmissions rates. The experience of the operator did not influence LOS $(p=0.201)$. Comparing each new operator with previous operator(s), the starting point in terms of duration of surgery was shorter, and the learning curve was steeper. The duration of surgery was significantly longer for supervised beginning operators as compared with experienced operators.

Conclusion Within the stepwise training program for residents, there is a slight increase in duration of surgery in the beginning of the learning curve, without affecting the patient outcome.
\end{abstract}

Keywords Learning curve $\cdot$ Roux-en-Y gastric bypass $\cdot$ Sleeve gastrectomy $\cdot$ ERABS $\cdot$ Enhanced recovery

$\begin{array}{ll}\text { Abbreviations } \\ \text { CI } & \text { Confidence interval } \\ \text { ERABS } & \text { Enhanced recovery after bariatric surgery } \\ \text { LOS } & \text { Length of stay } \\ \text { LRYGB } & \text { Laparoscopic Roux-en-Y gastric bypass } \\ \text { LSG } & \text { Laparoscopic sleeve gastrectomy } \\ \text { OR } & \text { Odds ratio } \\ \text { T2D } & \text { Type 2 diabetes } \\ \text { TSG } & \text { Time-subsequent group } \\ \text { VTE } & \text { Venous thromboembolic event }\end{array}$

M. Leeman

M.Leeman@Franciscus.nl

1 Department of Surgery, Franciscus Gasthuis \& Vlietland, Kleiweg 500, 3045 PM, Rotterdam, The Netherlands

2 Department of Surgery, Erasmus Medical Center, Rotterdam, The Netherlands

\section{Introduction}

Enhanced recovery after bariatric surgery (ERABS) protocols or fast-track protocols play a very important role in creating a clinical pathway that is both efficient and safe [1]. Short duration of surgery is an important aspect of the ERABS proto$\mathrm{col}$, as earlier research showed that there is a direct, inverse relation between duration of surgery and complication rates [2-4].

Several factors can influence the duration of surgery, including the level of experience of the operator [5, 6]. Training of surgical residents requires time-consuming education moments in the operating room and could influence duration of surgery, possibly affecting patient outcome and recovery after the procedure.

Over the years, many research groups have investigated the safety of resident involvement in high-risk surgical procedures such as bariatric surgery. Overall, all studies on this topic 
concluded that resident involvement in bariatric surgery is safe [5-10]. Little is known on resident involvement in fast-track bariatric surgery, in which perioperative efficiency is a key point contributing to safe early discharge.

The aim of this study is to evaluate the learning curve for the laparoscopic Roux-en-Y gastric bypass (LRYGB) and laparoscopic sleeve gastrectomy (LSG) of different operators who were educated in different time frames. Furthermore, the aim is to evaluate the impact of the operator's position on the learning curve on postoperative recovery outcome, in patients being treated according to the ERABS protocol. The study hypothesis is that the postoperative recovery is equal for all patients following the ERABS protocol, regardless of the operator's position on the learning curve.

\section{Methods}

\section{Design and Setting}

Data was collected from all patients who underwent a primary LRYGB or LSG, performed by operators that started and completed their training in bariatric surgery between 2004 and 2018 in a teaching hospital in the Netherlands. In the hospital's bariatric clinic, mainly LRYGBs and LSGs are performed. Exclusion criteria were a bariatric procedure combined with other surgical procedures, such as adhesiolysis, cholecystectomy or diaphragmatic hernia repair, or in case of conversion to an open procedure. Because of missing data on complications before implementation of the Dutch national complication registration database in 2014, analyses on postoperative complications were only performed for patients operated between 2014 and 2018 .

\section{Outcomes}

Primary outcome measure was duration of surgery, starting at placement of the Veress needle and ending after closing of all laparoscopic wounds. Secondary outcome measure was clinical outcome, in terms of length of hospital stay (LOS), minor and major complication rates, and readmission rates within 30 days postoperative. Type of complication was classified as described by Brethauer et al. [11].

\section{Training Program}

The operating techniques for LRYGB and LSG in our center have not changed significantly since 2004 and were described in previous publications $[12,13]$. Residents are trained to perform bariatric procedures using a stepwise LRYGB and LSG training program (Fig. 1), in which the surgeon in training starts with performing separated steps of the procedure instead of an entire procedure at once. During the training, an experienced surgeon was always present in the operating room or assisting in the procedure to be able to evaluate when a surgeon in training has become skilled enough to perform an entire procedure safely and in a timely manner. The results of the training program for the LRYGB are described by Walinga et al. [14].

\section{Statistical Analysis}

All analyses were performed using SPSS (PASW) 25 software (SPSS Inc., Chicago, Illinois, USA). For each operator, learning curves of both procedure types were created by dividing the procedures in time-subsequent groups (TSGs), with a maximum of 50 procedures per TSG. The learning curves were conducted based on the mean duration of surgery of the TSGs of each operator. The effect of the operator on the duration of surgery was evaluated using linear regression analysis. The first 50 LRYGBs and the first 50 LSGs that were performed entirely by the beginning operator were identified and compared with procedures performed by other operators in the same time frame. The procedures were compared on duration of surgery and length of hospital stay using one-way ANOVA. Operators who had performed less than 50 procedures were excluded from this analysis.

Data from 2014 to 2018 was analyzed by comparing "beginners" with "experienced operators" as first operator using multivariate logistic regression analysis, correcting for year of surgery, patient characteristics, and comorbidities (hypertension, type 2 diabetes (T2D), and dyslipidemia). After 100 procedures of one procedure type (either LRYGB or LSG), the operator was classified as "experienced" for that particular procedure type. Results were evaluated at a significance threshold of $p<0.05$ (two-sided).

\section{Results}

In total, 5137 primary procedures were performed between 2004 and 2018, of which 236 cases were primary bariatric procedures combined with other surgical procedures and therefore excluded. Table 1 shows the baseline characteristics by type of procedure of the 2411 (46.9\%) LRYGBs and 2726 $(53.1 \%)$ LSGs that were analyzed.

\section{Learning Curve per Individual Operator}

Figure 2 shows the mean duration of surgery in the first TSG (i.e., the first 50 procedures) of each operator for LRYGB (a) and LSG (b). The operators were put in chronological order by date of their first procedure. The mean duration of surgery decreased gradually with the start of each new operator for both procedure types, meaning that the starting point in terms of duration of surgery was shorter. Figure 3 illustrates the 
Fig. 1 Steps of the training program for LRYGB and LSG
Roux-en-Y gastric bypass

1. Creation of the gastric pouch

2. Identification of the ligament of Treitz, measuring the biliopancreatic limb and creating the stapled gastrojejunostomy

3. Laparoscopic suture closure of the linear stapled gastrojejunal anastomosis

4. Measuring the alimentary limb and creating the stapled jejunojejunostomy

5. Laparoscopic suture closure of the linear stapled jejunojejunal anastomosis
Sleeve gastrectomy

1. Dissection of omentum from the greater curvature of the stomach

2. Posterior mobilization of the stomach and angle of His

3. Ventral mobilization of angle of His

4. Stapling the stomach learning curve within the first 50 procedures of each operator. Duration of surgery was significantly shorter in the second half of the first TSG for LRYGB for all operators, and for LSG for only two operators.

In multivariate analysis correcting for year of surgery, patient characteristics, and comorbidities, there was no significant correlation between the operator and the duration of surgery $(\mathrm{OR}-0.210,95 \% \mathrm{CI}-0.915-0.496, p=0.557)$. The year of surgery (i.e., the experience of the operating team) was significantly correlated with the duration of surgery (OR $-3.353,95 \%$ CI $-6.070-0.635, p=0.016$ ).

Tables 2 and 3 show the duration of surgery and LOS (mean $\pm \mathrm{SD}$ ), respectively, for the first and second TSG of LRYGBs and LSGs of each individual operator. These means were compared with the duration of surgery and LOS performed by other operators in that same time frame (reference). No reference was available for the first operator, as this operator performed the first 50 procedures of both procedure types working as the only bariatric surgeon in this center and was therefore in the second TSG still the most experienced surgeon. After, for each operator and procedure type, the duration of the first 50 procedures was significantly longer compared with the reference. By the second TSG, one surgeon had equaled for duration of surgery with the reference operators for both LRYGB and LSG. Another surgeon had equaled the duration of surgery in the second TSG for only LSG. Most surgeons had shortened the duration of surgery but not yet equaled with the reference operators. Nevertheless, already in the first TSG, no difference was found in LOS between the beginning operator and the reference operators. There was a wide range of the time frame of the first 50 procedures of LRYGB and LSG between operators, varying from 4 months to 4 years.

\section{Beginning Versus Experienced Operator}

Tables 4 and 5 show the occurrence of complications and readmissions by level of experience for LRYGB and LSG, respectively. Chi-square analysis showed that the occurrence of minor complications, major complications, or readmissions was equal for both levels of experience of the surgeon. Multivariable analysis also showed that there was no significant difference between patients undergoing a LRYGB performed by a supervised beginner and an experienced operator

Table 1 Baseline characteristics

\begin{tabular}{lll}
\hline Characteristics & LRYGB $(n=2297)$ & LSG $(n=2604)$ \\
\hline Age at surgery (years), median, IQR & $43(34-50)$ & $41(32-49)$ \\
Female gender, $n(\%)$ & $1951(84.9 \%)$ & $2005(77.0 \%)$ \\
BMI at inclusion $\left(\mathrm{kg} / \mathrm{m}^{2}\right)$, mean \pm SD & $43 \pm 5$ & $43 \pm 5$ \\
Hypertension, $n(\%)^{*}$ & $391 / 1341(29.2 \%)$ & $243 / 1289(18.9 \%)$ \\
Diabetes, $n(\%) *$ & $245 / 1341(18.2 \%)$ & $177 / 1289(13.7 \%)$ \\
Dyslipidemia, $n(\%)^{*}$ & $203 / 1341(15.1 \%)$ & $168 / 1289(13.0 \%)$ \\
\hline
\end{tabular}

*42\% (LRYGB) and 50\% (LSG) missing data 
Fig. 2 a Mean duration of surgery (minutes) of the first TSG of LRYGBs per operator. b Mean duration of surgery (minutes) of the first TSG of LSGs per operator a

Mean duration of surgery (minutes) of the first

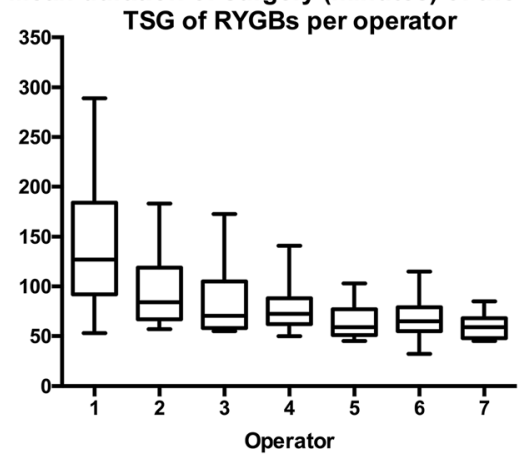

b

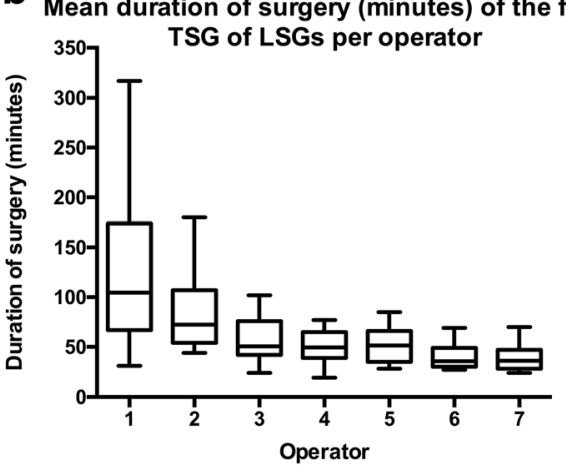

in minor complication rates (OR 0.712, 95\% CI 0.323-1.568, $p=0.399$ ), major complication rates (OR 1.217, 95\% CI $0.480-3.088, p=0.679$ ), or readmission rates (OR 0.974, 95\% CI $0.473-2.006, p=0.942)$. Similar results were found for LSG based on level of experience of the operator on minor complication rates (OR 0.835, 95\% CI 0.335-2.079, $p=$ 0.699 ), major complication rates (OR 4.148, OR 95\% CI $0.952-18.067, p=0.058$ ), or readmission rates (OR 1.154,
Fig. 3 Mean duration of surgery (minutes) comparing the first 25 and second 25 procedures per operator
Duration of surgery of the first 25 procedures and the second 25 procedures

\section{LRYGB}

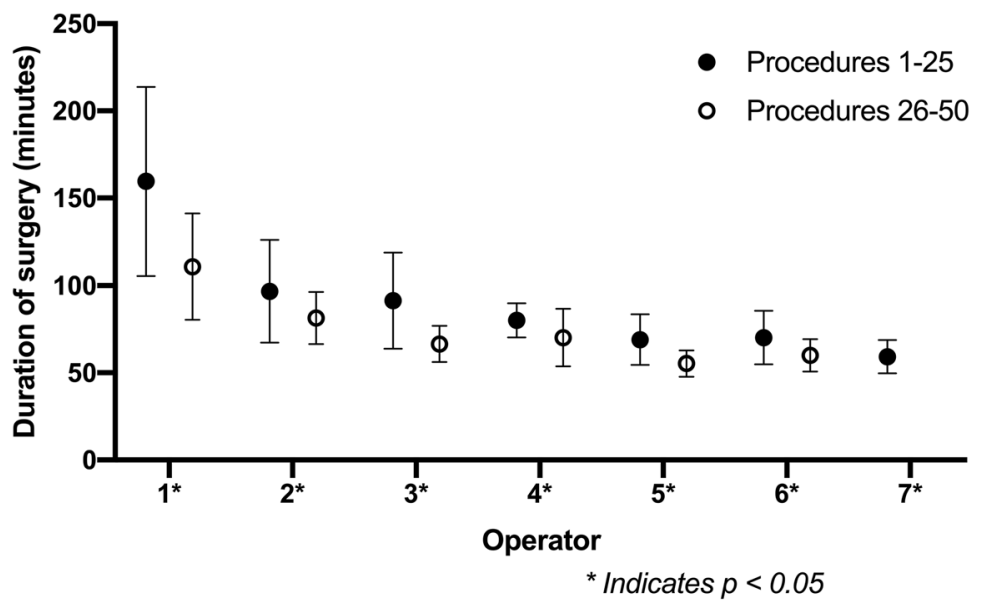

LSG

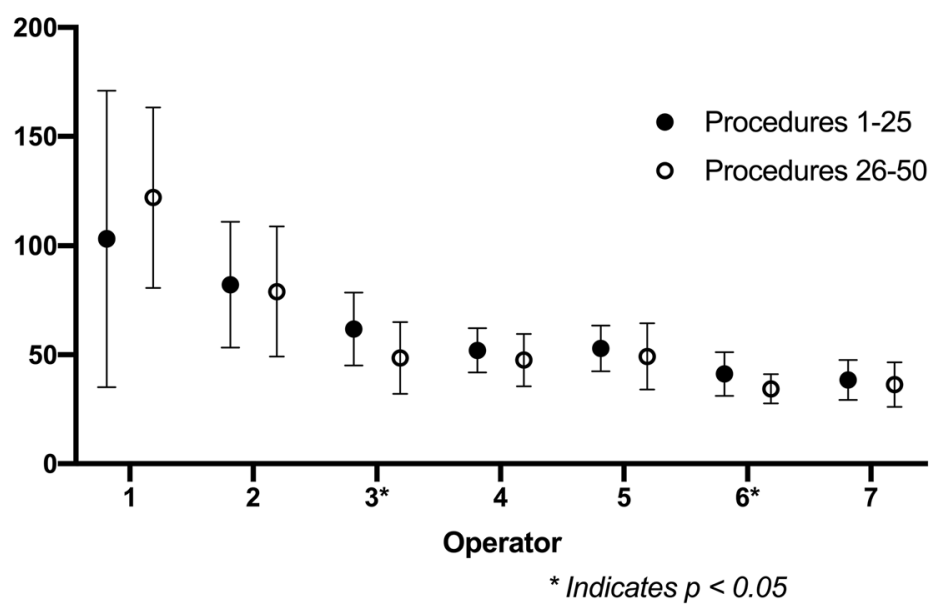


Table 2 Duration of surgery (mean $\pm \mathrm{SD}$ ) of the first and second TSG of LRYGBs and LSGs of each operator (number 1 to 7), compared with the duration of surgery in that time frame

\begin{tabular}{|c|c|c|c|c|c|c|c|}
\hline & \multirow[b]{2}{*}{ TSG } & \multicolumn{3}{|c|}{ Duration of LRYGB (minutes) } & \multicolumn{3}{|c|}{ Duration of LSG (minutes) } \\
\hline & & Operator & Reference & Sig. & Operator & Reference & Sig. \\
\hline \multirow[t]{2}{*}{$1 *$} & 1 & $135.22 \pm 49.96$ & - & - & $112.52 \pm 56.44$ & - & - \\
\hline & 2 & $70.98 \pm 23.82$ & - & - & $80.78 \pm 28.21$ & - & - \\
\hline \multirow[t]{2}{*}{2} & 1 & $89.10 \pm 24.33$ & $60.31 \pm 18.56$ & $<0.001$ & $81.24 \pm 29.11$ & $65.89 \pm 24.31$ & 0.002 \\
\hline & 2 & $73.21 \pm 13.93$ & $53.79 \pm 17.65$ & $<0.001$ & $66.29 \pm 19.20$ & $50.78 \pm 19.70$ & $<0.001$ \\
\hline \multirow[t]{2}{*}{3} & 1 & $81.65 \pm 24.95$ & $70.39 \pm 28.72$ & 0.022 & $55.37 \pm 18.10$ & $47.29 \pm 18.37$ & 0.006 \\
\hline & 2 & $65.68 \pm 17.07$ & $67.17 \pm 14.55$ & 0.657 & $40.64 \pm 10.47$ & $45.43 \pm 13.23$ & 0.060 \\
\hline \multirow[t]{2}{*}{4} & 1 & $75.45 \pm 14.37$ & $52.25 \pm 16.78$ & $<0.001$ & $49.90 \pm 11.06$ & $38.90 \pm 12.75$ & $<0.001$ \\
\hline & 2 & $67.25 \pm 18.34$ & $52.45 \pm 16.82$ & $<0.001$ & $48.10 \pm 14.32$ & $35.86 \pm 8.44$ & $<0.001$ \\
\hline \multirow[t]{2}{*}{$5 * *$} & 1 & $61.94 \pm 13.35$ & $52.67 \pm 16.47$ & $<0.001$ & $50.73 \pm 13.14$ & $38.17 \pm 11.54$ & $<0.001$ \\
\hline & 2 & $50.97 \pm 8.16$ & $46.04 \pm 13.33$ & 0.030 & $36.13 \pm 9.20$ & $33.05 \pm 9.07$ & 0.019 \\
\hline \multirow[t]{2}{*}{$6 * * *$} & 1 & $66.24 \pm 14.13$ & $48.64 \pm 13.68$ & $<0.001$ & $37.65 \pm 9.09$ & $36.33 \pm 11.02$ & 0.404 \\
\hline & 2 & - & - & - & - & - & - \\
\hline \multirow[t]{2}{*}{$7 * * *$} & 1 & $59.25 \pm 9.57$ & $44.80 \pm 8.56$ & $<0.001$ & $37.25 \pm 9.53$ & $31.24 \pm 7.31$ & $<0.001$ \\
\hline & 2 & - & - & - & - & - & - \\
\hline
\end{tabular}

95\% CI 0.510-2.611, $p=0.731$ ). Considering the type of procedure and the year of surgery, the experience of the operator did not influence the LOS $(p=0.201)$.

\section{Discussion}

The aim of this study was to evaluate the impact of the level of experience of the operator on postoperative recovery, in patients being treated according to the ERABS protocol. We found that the experience of the operator is negatively correlated with the duration of surgery. In this report, we show that although duration of surgery is longer in the learning curve period this does not affect postoperative recovery or complication rates. Therefore, we can conclude that it is safe to involve a resident training program in a fast-track setting.

\section{Duration of Surgery}

A gradual decrease was seen in the mean duration of surgery in the first TSG with the start of each new operator for both procedures. This can be explained by the fact that over the years, the mean duration of surgery shortened due to increasing experience of the dedicated bariatric team [15]. Therefore, each new operator started their education in an operating team that had already progressed in their own learning curves. Furthermore, education in the operating room became easier after introduction of the stepwise learning program for LRYGB and LSG in 2015 [14].

Within the first TSG, duration of surgery was significantly shorter in the second half of the TSG for LRYGB for all operators, and for LSG for only two out of seven. This result demonstrates that the learning curve of the LRYGB is steeper than the learning curve of the LSG. Two theories can be formed on this matter. The first theory holds that steepness of the learning curve
Table 3 Length of hospital stay (median \pm IQR) of the first TSG of LRYGBs and LSGs of each operator (number 1 to 7 ), compared with the length of hospital stay in that time frame

\begin{tabular}{|c|c|c|c|c|c|c|}
\hline & \multicolumn{3}{|c|}{ Length of hospital stay after LRYGB (days) } & \multicolumn{3}{|c|}{ Length of hospital stay after LSG (days) } \\
\hline & Operator & Reference & Sig. & Operator & Reference & Sig. \\
\hline $1 *$ & $3.18 \pm 0.99$ & - & - & $4.46 \pm 2.04$ & - & - \\
\hline 2 & $2.00 \pm 1.83$ & $2.00 \pm 1.00$ & 0.903 & $3.09 \pm 1.12$ & $3.17 \pm 1.02$ & 0.775 \\
\hline 3 & $2.52 \pm 1.05$ & $2.18 \pm 0.94$ & 0.966 & $3.05 \pm 0.99$ & $3.01 \pm 1.00$ & 0.064 \\
\hline 4 & $1.20 \pm 0.80$ & $1.21 \pm 0.22$ & 0.399 & $1.29 \pm 0.96$ & $1.28 \pm 0.34$ & 0.433 \\
\hline $5 * *$ & $1.15 \pm 0.12$ & $1.16 \pm 0.15$ & 0.436 & $1.15 \pm 0.20$ & $1.21 \pm 0.74$ & 0.001 \\
\hline $6 * * *$ & $1.15 \pm 0.17$ & $1.15 \pm 0.15$ & 0.344 & $1.13 \pm 0.25$ & $1.17 \pm 0.28$ & 0.323 \\
\hline $7 * * *$ & $1.16 \pm 0.11$ & $1.15 \pm 0.14$ & 0.840 & $1.12 \pm 0.18$ & $1.18 \pm 0.17$ & 0.004 \\
\hline
\end{tabular}

*No reference data available

**Operator performed less than 100 procedures in total

*** Operator performed less than 50 procedures in total 
Table 4 Occurrence of complications and readmissions by level of experience for LRYGB

\begin{tabular}{llll}
\hline LRYGB & Beginning surgeon & Experienced surgeon & Sig. \\
\hline Minor complications $(n, \%)$ & $12 / 304(3.9 \%)$ & $34 / 1226(2.8 \%)$ & 0.283 \\
Major complications $(n, \%)$ & $10 / 304(3.3 \%)$ & $36 / 1226(2.9 \%)$ & 0.747 \\
Readmissions $(n, \%)^{*}$ & $15 / 292(5.1 \%)$ & $47 / 1173(4.0 \%)$ & 0.391 \\
\hline
\end{tabular}

*Missing data on readmissions for LRYGB (4.2\%) is a positive factor, meaning that the operator is quickly improving in the learning process. This would imply that the LRYGB is easier to learn than the LSG. The second theory, which we support, holds that a non-steep learning curve can imply that a procedure is easier to learn; as from the beginning, duration of surgery is relatively short.

There was a wide range in TSGs of 4 months -4 years, which can be explained by the fact that some of the operators started performing bariatric procedures during their residency. During their residency, they followed different internships which were spread out into different hospitals. However, they have only performed bariatric procedures in this center, ensuring that their learning curve was not pursued during their absence.

The mean duration of surgery of the first 50 procedures was longer for each operator, in comparison with procedures performed by other operators in the same time frame. The extension of the procedure due to operating by a beginning operator was significant, yet small (15-30 min for LRYGB, 6-16 min for LSG). When working in a fast-track setting, the time that the anesthesiological team needs for induction and emergence is short. Therefore, the extra time a beginning operator might need has a relatively large effect on the total duration of surgery and is therefore significantly different. However, the absolute amount of additional time is not excessive. Chan et al. and Krell et al. stated that duration of surgery was an independent predictor of postoperative venous thromboembolic events (VTE) $[2,16]$. A recently published article from our research group based on the same database revealed that the clinical VTE rates in this center have been very low since $2014(<1 \%)$ [17], showing that training residents have not led to increased VTE rates.

\section{Length of Hospital Stay}

Earlier research has commented on the prolonged hospital stay of patients that were operated with resident involvement [18].
In this study, the level of experience of the operator did not influence the $\operatorname{LOS}(p=0.201)$. The mean difference of 0.71 days between beginning and experienced operators seems substantial, but can easily be explained by the fact that the majority of the analyzed procedures that were performed by beginners took place before introduction of ERABS. As earlier research has shown, the LOS has significantly reduced after the introduction of ERABS in our center [15].

\section{Morbidity}

A great concern with respect to resident involvement in bariatric surgery is the possible increase in morbidity due to insufficient experience of the operator. Several studies reported increased morbidity rates, but mainly in minor complications $[7,16,19,20]$. In this study, there was no difference between patients undergoing a LRYGB or LSG performed by a beginner and an experienced operator in minor complication rates, major complication rates, or readmission rates. Similar results in the research area of upper gastrointestinal surgery were found by Philips et al., describing that patient outcomes are not compromised by supervised trainee involvement in transthoracic esophagectomy [21].

\section{Limitations}

A limitation of this study was that the baseline surgical experience of the operator was not taken into account. This was due to the lack of data on the number of bariatric procedures the surgeon or surgical resident had assisted and the years of additional experience as a resident and/or surgeon. Also, before performing a procedure entirely, the beginning surgeon has performed steps of the procedure while assisting the experienced surgeon. Herewith, the learning curve might have started before the first TSG. Unfortunately, it was impossible to determine which steps were performed by the beginner in each procedure from this retrospective data. Nevertheless, this
Table 5 Occurrence of complications and readmissions by level of experience for LSG

\begin{tabular}{llll}
\hline LSG & Beginning surgeon & Experienced surgeon & Sig. \\
\hline Minor complications $(n, \%)$ & $4 / 284(1.4 \%)$ & $41 / 1158(3.5 \%)$ & 0.064 \\
Major complications $(n, \%)$ & $10 / 284(3.5 \%)$ & $27 / 1158(2.3 \%)$ & 0.256 \\
Readmissions $(n, \%) *$ & $12 / 259(4.6 \%)$ & $42 / 1111(3.7 \%)$ & 0.525 \\
\hline
\end{tabular}

*Missing data on readmissions for LSG (5.0\%) 
data suggests that the learning curve for LRYGB or LSG and the patient outcome are mainly determined by level of experience of the surgical team that is teaching the procedure. Therefore, baseline surgical experience seems to have no additional value.

\section{Conclusion}

The level of experience of the surgeon did not influence patient complication rates or length of hospital stay. Within the stepwise training program for residents, there is a slight increase in duration of surgery in the beginning of the learning curve. Training residents is an essential task for all surgical units, including the bariatric unit. This study showed that resident involvement and peroperative training according to a stepwise learning program could be encouraged in bariatric surgery.

\section{Compliance with Ethical Standards}

Conflict of Interest The authors declare that they have no conflict of interest.

Ethical Approval All procedures performed in studies involving human participants were in accordance with the ethical standards of the institutional and/or national research committee and with the 1964 Helsinki Declaration and its later amendments or comparable ethical standards.

Informed Consent Informed consent was obtained from all individual participants included in the study.

\section{References}

1. Thorell A, MacCormick AD, Awad S, et al. Guidelines for perioperative care in bariatric surgery: enhanced recovery after surgery (ERAS) society recommendations. World J Surg. 2016;40(9): 2065-83.

2. Chan MM, Hamza N, Ammori BJ. Duration of surgery independently influences risk of venous thromboembolism after laparoscopic bariatric surgery. Surgery for obesity and related diseases : official journal of the American Society for Bariatric Surgery. 2013;9(1):88-93.

3. Major P, Wysocki M, Pedziwiatr M, et al. Risk factors for complications of laparoscopic sleeve gastrectomy and laparoscopic Rouxen-Y gastric bypass. International Journal of Surgery (London, England). 2017;37:71-8.

4. Nandipati K, Lin E, Husain F, et al. Factors predicting the increased risk for return to the operating room in bariatric patients: a NSQIP database study. Surg Endosc. 2013;27(4):1172-7.

5. D'Souza N, Hashimoto DA, Gurusamy K, et al. Comparative outcomes of resident vs attending performed surgery: a systematic review and meta-analysis. Journal of surgical education. 2016;73(3):391-9.
6. Major P, Wysocki M, Dworak J, et al. Are bariatric operations performed by residents safe and efficient? Surgery for obesity and related diseases : official journal of the American Society for Bariatric Surgery. 2017;13(4):614-21.

7. Doyon L, Moreno-Koehler A, Ricciardi R, et al. Resident participation in laparoscopic Roux-en-Y gastric bypass: a comparison of outcomes from the ACS-NSQIP database. Surg Endosc. 2016;30(8):3216-24.

8. Iordens GI, Klaassen RA, van Lieshout EM, et al. How to train surgical residents to perform laparoscopic Roux-en-Y gastric bypass safely. World J Surg. 2012;36(9):2003-10.

9. Kuckelman J, Bingham J, Barron M, et al. Advanced laparoscopic bariatric surgery is safe in general surgery training. Am J Surg. 2017;213(5):963-6.

10. Rovito PF, Kreitz K, Harrison TD, et al. Laparoscopic Roux-en-Y gastric bypass and the role of the surgical resident. Am J Surg. 2005;189(1):33-7.

11. Brethauer SA, Kim J, el Chaar M, et al. Standardized outcomes reporting in metabolic and bariatric surgery. Surgery for obesity and related diseases : official journal of the American Society for Bariatric Surgery. 2015;11(3):489-506.

12. Biter LU, Gadiot RP, Grotenhuis BA, et al. The sleeve bypass trial: a multicentre randomized controlled trial comparing the long term outcome of laparoscopic sleeve gastrectomy and gastric bypass for morbid obesity in terms of excess BMI loss percentage and quality of life. BMC obesity. 2015;2:30.

13. Gadiot RP, Biter LU, Zengerink HJ, et al. Laparoscopic sleeve gastrectomy with an extensive posterior mobilization: technique and preliminary results. Obes Surg. 2012;22(2):320-9.

14. Walinga AB, van Mil SR, Biter LU, et al. A stepwise approach in learning surgical residents a Roux-en-Y gastric bypass. Obes Surg. 2018;

15. Mannaerts GH, van Mil SR, Stepaniak PS, et al. Results of implementing an enhanced recovery after bariatric surgery (ERABS) protocol. Obes Surg. 2016;26(2):303-12.

16. Krell RW, Birkmeyer NJ, Reames BN, et al. Effects of resident involvement on complication rates after laparoscopic gastric bypass. J Am Coll Surg. 2014;218(2):253-60.

17. Leeman M, Biter LU, Apers JA, et al. A single-center comparison of extended and restricted thromboprophylaxis with LMWH after metabolic surgery. Obesity surgery. 2019; In press.

18. Carter J, Elliott S, Kaplan J, et al. Predictors of hospital stay following laparoscopic gastric bypass: analysis of 9,593 patients from the National Surgical Quality Improvement Program. Surgery for obesity and related diseases : official journal of the American Society for Bariatric Surgery. 2015;11(2):288-94.

19. Major P, Wysocki M, Dworak J, et al. Analysis of laparoscopic sleeve gastrectomy learning curve and its influence on procedure safety and perioperative complications. Obes Surg. 2018;28(6): 1672-80.

20. Zacharoulis D, Sioka E, Papamargaritis D, et al. Influence of the learning curve on safety and efficiency of laparoscopic sleeve gastrectomy. Obes Surg. 2012;22(3):411-5.

21. Phillips AW, Dent B, Navidi M, et al. Trainee involvement in Ivor Lewis esophagectomy does not negatively impact outcomes. Ann Surg. 2018;267(1):94-8.

Publisher's Note Springer Nature remains neutral with regard to jurisdictional claims in published maps and institutional affiliations. 